\title{
Analyse de poste : contamination atmosphérique au tritium
}

\author{
S. RIGAUD ${ }^{1}$, S. CHANAL ${ }^{2}$, B. MAYNADIER ${ }^{2}$, F. LEMONTEY ${ }^{1}$, \\ C. LECRIQUE-GELHAY ${ }^{1}$, F. GAUDET ${ }^{3}$, V. RAUFAST ${ }^{4}$, O. COLAS $^{3}$
}

(Manuscrit reçu le 24 juin 2011, accepté le 2 décembre 2011)

RÉSUMÉ La contamination radioactive, qu'elle soit de nature surfacique ou atmosphérique, peut être la cause d'une exposition interne de l'individu. Les valeurs Limites pratiques de concentration dans l'air (LPCA) permettent aux médecins du travail ainsi qu'aux Personnes compétentes en Radioprotection (PCR) de pré-évaluer les risques résultant d'une contamination atmosphérique. Ces valeurs sont utilisées pour déterminer la conduite à tenir au poste de travail mais aussi comme outil d'optimisation de la protection des personnes dans le cadre de l'application du principe ALARA (As Low As Reasonably Achievable). Lors des analyses de poste, les PCR des laboratoires Pierre Fabre ont été confrontées à des valeurs de dose efficace engagée qui semblaient, de leur point de vue, anormalement élevées. Ces valeurs étaient en contradiction avec les résultats des examens de radiotoxicologie urinaire réalisés dans le cadre de la surveillance médicale renforcée des manipulateurs, qui se sont toujours avérés négatifs (que ces examens soient effectués périodiquement ou de façon ponctuelle à la fin d'une série de manipulations radioactives). C'est pourquoi il a été jugé pertinent de louer des systèmes de barboteurs, équipements utilisés pour la surveillance des faibles niveaux de concentration en tritium et carbone 14. Les mesures réalisées ont permis de mettre en évidence des niveaux de contamination atmosphérique en tritium négligeables, notamment lors des étapes de manipulation jugées a priori pénalisantes. Ce travail, mené dans le cadre des études de poste, nous a permis d'abaisser la valeur du facteur de volatilité des composés tritiés intervenant dans le calcul de dose efficace engagée.

ABSTRACT Analysis of workstation: tritium atmospheric contamination.

Radioactive contamination, whether it is on the surface or in the atmosphere, could be the reason for individual internal exposure. The Practical Air Contamination Limit values enable the occupational health doctors as well as the "Personne Compétente en Radioprotection" (PCR: competent person in radioprotection) to pre-evaluate the risks resulting from atmospheric contamination. These values are used to determine the course of action regarding the workstation, but also as an optimisation tool for staff protection, within the framework of the application of the ALARA (As Low As Reasonably Achievable) principal. During the analysis of workstations, the PCRs at Pierre Fabre Laboratories were confronted with effective dose values which seemed to be, in their opinion, abnormally high. These values were in contradiction with the results of the urinary radiotoxicological exams, which are

\footnotetext{
${ }^{1}$ Centre de Recherche Pierre Fabre, 17 avenue Jean Moulin, 81100 Castres.

${ }^{2}$ Centre Expérimental et Pharmacocinétique Pierre Fabre, 81100 Castres.

${ }^{3}$ Centre d'Immunologie Pierre Fabre, 5 avenue Napoléon III, 74160 Saint-julien-en-Genevois.

${ }^{4}$ Centre de Recherche et Développement Pierre Fabre, 3 avenue Hubert Curien, 31560 Toulouse.
} 


\begin{abstract}
done within the framework of the reinforced medical monitoring of the technicians, and which have always been negative (whether the exams were done periodically or from time to time at the end of a radioactive experiment series). This is why it was considered relevant to rent bubble chamber systems, used for low-level concentrations of tritium and carbon-14. The measurements showed insignificant tritium atmospheric contamination levels in the laboratories, in particular for some experimental steps that were considered a priori problematic. This study, carried out within the framework of the workstation, enabled us to decrease the volatility factor value of tritiated compounds intervening in the effective dose calculation.
\end{abstract}

Keywords: Effective dose / atmospheric contamination / tritium / volatility factor

\title{
1. Introduction
}

\subsection{Utilisation des sources radioactives}

Certaines activités de recherche, et particulièrement la recherche en biologie moléculaire et en métabolisme in vivo, font appel à des sources radioactives. Il est, par exemple, possible de marquer certaines molécules pour suivre leur évolution au sein d'une cellule ou d'un organisme vivant. L'utilisation de traceurs radioactifs incorporés à des molécules est un outil puissant d'investigation en biologie cellulaire et moléculaire, en pharmacocinétique et métabolisme. Les principaux radio-isotopes utilisés sous forme non scellée sont le phosphore 32 ou 33, le carbone 14 , le soufre 35 , le chrome 51, l'iode 125 et le tritium.

Les activités de produits radioactifs généralement utilisées sont relativement faibles ; malgré tout l'utilisation de ces radionucléides sous forme non scellée peut entraîner un risque de contamination interne du personnel par inhalation.

Il est à noter que, dans de très nombreux cas, les distributeurs de sources radioactives (pourtant référencés par les autorités compétentes) ne fournissent aucune information relative à la volatilité des produits radiomarqués qu'ils proposent.

Nous allons lors de cette étude nous attarder sur le cas du tritium, car il s'agit d'un radionucléide très utilisé dans nos laboratoires et difficilement détectable. En effet, le tritium est un émetteur $\beta$ ayant une énergie moyenne de 5,68 $\pm 0,01 \mathrm{keV}$, une période de 12,312 \pm 0,025 ans (Baglan et al., 2010 ; LNE-LNHB/CEA, 2006).

\subsection{Caractérisation des risques}

En tant qu'isotope de l'hydrogène, le tritium ne présente pas de toxicité chimique (au contraire de l'uranium, par exemple). La radioactivité du tritium se résume à une émission du rayonnement bêta faiblement énergétique qui n'induit pas de risque d'exposition externe. La contamination, si elle existe, est toujours interne et 
les voies d'absorption sont l'inhalation, l'ingestion et la diffusion et/ou lésion cutanée. Les risques liés au tritium gazeux sont plus de 10000 fois plus faibles que ceux liés au tritium sous la forme d'eau tritiée. En cas d'émission d'un mélange d'hydrogène tritié (HT) et d'eau tritiée (HTO) (c'est pratiquement toujours le cas), la contamination sera directe par HTO. En revanche, pour HT, le mécanisme sera d'abord une oxydation par les bactéries de l'interface sol-atmosphère puis une libération d'eau tritiée dans l'atmosphère (Belot et al., 1996).

Quantitativement, on peut retenir que la dose efficace par unité d'incorporation pour le tritium est néanmoins l'une des plus faibles de tous les radionucléides (Belot et al., 1996). Rappelons que « plus faible » veut dire, ici, dans un rapport de plusieurs ordres de grandeur.

Le métabolisme du tritium après incorporation dépend de sa forme moléculaire :

$-\mathrm{HTO}$

- HT;

- tritium organiquement lié (TOL) : méthane, hydrures métalliques, solvants, huiles, molécules marquées utilisées en recherche ou comme radiopharmaceutiques...

Le métabolisme des molécules organiques marquées dépend de la structure chimique et de la place de ${ }^{3} \mathrm{H}$ dans la molécule.

\section{Problématique : évaluation de l'exposition interne ou dose efficace engagée - Justification de la valeur du coefficient de volatilité $K$}

\subsection{Le contexte réglementaire}

La notion d'étude de poste apparaît à l'article R. 4451-11 du code du travail où il est précisé : «Dans le cadre de l'évaluation des risques, l'employeur [...] procède à une analyse des postes de travail qui est renouvelée périodiquement et à l'occasion de toute modification des conditions pouvant affecter la santé et la sécurité des travailleurs. »

Afin d'estimer la contamination interne par inhalation des manipulateurs et de comparer ces valeurs aux limites d'exposition fixées aux articles D. 4152-5, D. 4153-34, R. 4451-12 et R. 4451-13 du code du travail, nous avons choisi d'évaluer plus particulièrement le risque de contamination atmosphérique par le tritium. 
Le but de cette étude a été de rechercher et de quantifier, si nécessaire, la présence de radioactivité dans l'air potentiellement inhalable par le manipulateur lors des étapes les plus pénalisantes, afin d'estimer de manière plus précise la valeur du facteur de volatilité (K) pour chaque type d'étude de poste. Évaluer le risque d'exposition externe et interne après incorporation d'un radionucléide, permet de calculer la dose efficace engagée pour chaque manipulation et pour le cumul des manipulations réalisées sur une année. Ce cumul est comparé aux limites réglementaires pour définir le classement des travailleurs soumis aux rayonnements ionisants.

\section{2. Évaluation de l'exposition interne}

La plupart des radio-isotopes de faible énergie utilisés en recherche ne permettent pas d'obtenir une évaluation d'exposition empirique, l'utilisation de dosimètres passifs ou actifs n'étant pas adaptée.

La dose efficace susceptible d'être engagée suite à une exposition interne est évaluée en tenant compte de la nature et des caractéristiques des radionucléides susceptibles d'être présents sur un temps légal de travail annuel. Ce calcul est réalisé aussi bien en situation normale de travail qu'en situation dégradée.

Pour cette évaluation, nous avons utilisé, dans un premier temps, une formule très pénalisante du point de vue des niveaux d'exposition potentielle, en faisant les approximations suivantes :

- chaque tâche nécessitant la manipulation d'une source de tritium induit un risque d'inhalation en conditions normales de travail ;

- les facteurs temps, débit respiratoire et volume du local ne sont pas pris en compte au niveau du calcul ;

- pour l'évaluation de la dose interne reçue pour chaque tâche effectuée, l'activité mise en jeu est considérée comme étant égale à l'activité totale manipulée.

La formule est la suivante : $E_{\text {int }}=A \times K \times(1 / f) \times D P U I$

Avec : $\quad E_{\text {int }}=$ Estimation de la dose efficace engagée

$A=$ Activité manipulée $(\mathrm{Bq})$

$K=$ coefficient de volatilité du composé radioactif (sans dimension) obtenu à partir des fiches issues du Guide pratique « Radionucléides et Radioprotection» (Delacroix et al., 2006). 
Ce facteur $K$ représente alors le pourcentage de l'activité mise en œuvre potentiellement inhalable par le manipulateur.

Par défaut, on applique un facteur $K$ de 0,01 qui sera pondéré en fonction de la forme physico-chimique de la source, du solvant de dilution, des données fournisseurs et de notre propre expérience.

$$
\begin{aligned}
& f=\begin{array}{l}
\text { facteur de sécurité lié aux dispositifs de protection collective utilisés lors de } \\
\text { la manipulation }
\end{array} \\
& f: \quad \text { sur paillasse }=1 \\
& \quad \text { sous enceinte ventilée }=10 \\
& \quad \text { sous boîte à gants }=1000 .
\end{aligned}
$$

$D P U I=h(g)=$ Dose par unité d'incorporation du radionucléide concerné due à l'inhalation (en Sv.Bq-1).

La valeur de DPUI est donnée par l'annexe de l'arrêté du $1^{\mathrm{er}}$ septembre 2003 définissant les modalités de calcul des doses efficaces et des doses équivalentes résultant de l'exposition des personnes aux rayonnements ionisants. Plusieurs valeurs peuvent exister pour un même radionucléide sous des formes chimiques différentes. En cas de doute sur la forme chimique, la valeur la plus restrictive est à prendre en considération en l'absence d'informations plus précises sur les aérosols en cause.

La classification des travailleurs se basant sur des conditions normales de travail, on considérera qu'il n'y a pas d'ingestion de radionucléide.

Pour nos calculs de doses efficaces engagées, on considère généralement que l'inhalation est la voie d'incorporation la plus probable d'où le choix d'utiliser la

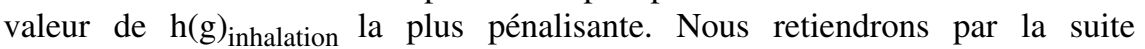
$h(\mathrm{~g})_{\text {inhalation }}=4,110^{-11} \mathrm{~Sv} \cdot \mathrm{Bq}^{-1}$ pour le ${ }^{3} \mathrm{H}$.

La formule $« E_{\text {int }}=A \times K \times(1 / f) \times h(g)_{\text {inhalation }}$ " engendre pour des activités de départ de l'ordre de $140 \mathrm{MBq}$ (correspondant à une seule étude), des valeurs très pénalisantes proches de la limite d'un travailleur classé en catégorie A.

Ces valeurs aberrantes nous ont très vite conduit à utiliser une formule basée sur l'activité volumique $A v$ susceptible d'être présente au poste de travail afin d'en déduire l'activité susceptible d'être inhalée, $A_{\mathrm{inh}}$.

$$
A_{\text {inh }}=A v \times Q \times t
$$


Avec $A v\left(\right.$ Bq. $\left.\mathrm{m}^{-3}\right)$ : activité volumique dans l'air du laboratoire $Q\left(\mathrm{~m}^{3} \cdot \mathrm{h}^{-1}\right):$ débit respiratoire de l'individu au travail $\left(1,2 \mathrm{~m}^{3} \cdot \mathrm{h}^{-1}\right)$

$t$ (h) : temps d'exposition.

Pour estimer la dose efficace engagée $E_{\text {int }}$ en Sv, l'activité susceptible d'être inhalée est multipliée par la valeur de $h(g)_{\text {inhalation }}$, selon :

$$
E_{\mathrm{int}}=A_{\mathrm{inh}} \times h(g)_{\text {inhalation }}
$$

Ce qui revient également à exprimer $E_{\text {int }}$ suivant la relation suivante :

$$
E_{\text {int }}=A v \times Q \times t \times h(g)_{\text {inhalation }}=\frac{A \times k}{V \times f} \times Q \times t \times h(g)_{\text {inhalation }}
$$

Avec cette nouvelle approche, nous obtenons pour une seule étude des valeurs proches de la limite d'un travailleur classé en catégorie B et non plus en catégorie A.

Bien que moins pénalisants, les derniers résultats obtenus restent encore éloignés de nos propres convictions. En effet, l'ensemble des contrôles de radiotoxicologie urinaire effectués soit à la fin d'une série de manipulations, soit au maximum à la fin de chaque semestre se sont avérés négatifs depuis de nombreuses années.

Face aux résultats précédemment obtenus, la question suivante nous parut évidente : Ne surestime-t-on pas la valeur du facteur $K$ correspondant à la fraction d'activité potentiellement inhalé ?

Afin de répondre au mieux à cette problématique, nous avons loué un système de barboteurs capable de mesurer, en temps réel, l'activité volumique de l'air dans nos laboratoires. Ceci dans le but de confronter théorie et résultats expérimentaux.

Concernant le classement du personnel, les Limites pratiques de contamination dans l'air (lpca exprimée en Bq. ${ }^{-3}$ ) calculées dans le Guide pratique «Radionucléides et Radioprotection »(Delacroix et al., 2006) peuvent également être utilisés, mais dans ce cas il faut se rappeler que ces LPCA sont calculées avec temps d'exposition de 2000 heures en prenant en compte l'exposition interne par inhalation et l'exposition externe par immersion dans l'atmosphère contaminée (Radecki et al., 2007).

LPCA ${ }^{3} H=2 \mathrm{E}+05 \mathrm{~Bq} \cdot \mathrm{m}^{-3}$ (conduisant à une dose efficace de $20 \mathrm{mSv}$ ) ;

LPCA ${ }^{3} H=1 \mathrm{E}+04 \mathrm{~Bq} \cdot \mathrm{m}^{-3}$ (conduisant à une dose efficace de $1 \mathrm{mSv}$ ). 
Quant au classement du local, lorsque la zone présente un risque d'exposition interne, le zonage se fera en fonction des mesures ou du calcul de contamination atmosphérique. On peut alors comparer les résultats obtenus, extrapolés à une durée de manipulation d'une heure à une autre grandeur pratique : le RCA ou Repère en Contamination Atmosphérique exprimé en Bq. $\mathrm{m}^{-3}$.

$$
\begin{gathered}
\mathrm{RCA}^{3} H \cong 5 E+05 \text { Bq. } \mathrm{m}^{-3} \text { (limite supérieure de la zone contrôlée, } \\
\text { soit } 25 \mu \mathrm{Sv} \text { en } 1 \mathrm{~h}) ; \\
\mathrm{RCA}^{3} H \cong 1,5 E+05 \text { Bq. }{ }^{-3} \text { (limite supérieure de la zone surveillée, } \\
\text { soit } 7,5 \mu \mathrm{Sv} \text { en } 1 \mathrm{~h}) .
\end{gathered}
$$

\section{Matériel et méthodes}

\subsection{Matériel}

Appareil de prélèvement de tritium atmosphérique (barboteur du type SDEC MARC 7000).

Ce système de prélèvement est principalement utilisé pour la surveillance des faibles niveaux de concentration en tritium atmosphérique sous forme vapeur provenant des cheminées, des systèmes de ventilation, des hottes, des locaux ou dans l'environnement. Cet appareil s'intègre parmi les instruments de surveillance de la filière nucléaire : centrales nucléaires de production d'électricité, centres de recherche nucléaire, sites de stockage et traitement des déchets radioactifs, laboratoires, incinérateurs, phase de démantèlement ou toutes industries intégrant du tritium.

\section{Principe de fonctionnement}

L'appareil prélève l'air à un débit déterminé (environ 30 litres/heure), pendant une durée choisie. Après filtration des aérosols de l'air, le MARC 7000 piège la vapeur d'eau tritiée (HTO) par le principe de barbotage dans l'eau contenue dans les biberons. Le gaz tritium est quant à lui oxydé sous forme de vapeur dans un four à catalyseur puis piégé par le principe de barbotage dans les deux derniers biberons. Après le temps de barbotage préalablement choisi, la quantité de tritium contenue dans l'eau des récipients de collecte est mesurée en laboratoire par comptage en scintillation liquide. L'activité totale du tritium déterminée est rapportée au volume d'air passé dans l'appareil. 


\section{Système de refroidissement de la solution de piégeage}

L'appareil est équipé d'un circuit froid qui permet de maintenir la solution de piégeage à une température moyenne de $7{ }^{\circ} \mathrm{C}$ (pour une température ambiante de $20^{\circ} \mathrm{C}$ ) et d'éviter ainsi une perte de la solution échantillon par évaporation. Cette fonction est indispensable pour un prélèvement hebdomadaire ou de plus longue durée.

\section{Circuit d'air}

Un filtre papier qui arrête les poussières à l'admission d'air (diamètre : $45 \mathrm{~mm}$ ).

Un débitmètre électronique de type massique protégé en entrée et sortie par des filtres Goretex.

\section{Performances du MARC 7000}

Rendement de piégeage en HTO : $99 \% \pm 7 \%$ *

Rendement de conversion du four en HTO : $98 \% \pm 7 \%$ *

Reproductibilité du débit d'air : écart maxi 0,8\%

Justesse du débit d'air : écart maxi $1 \%$

Réponse aux autres gaz radioactifs : influence nulle.

* : compte-rendu d'essai du CEA Valrhô (Marcoule), juin 2006

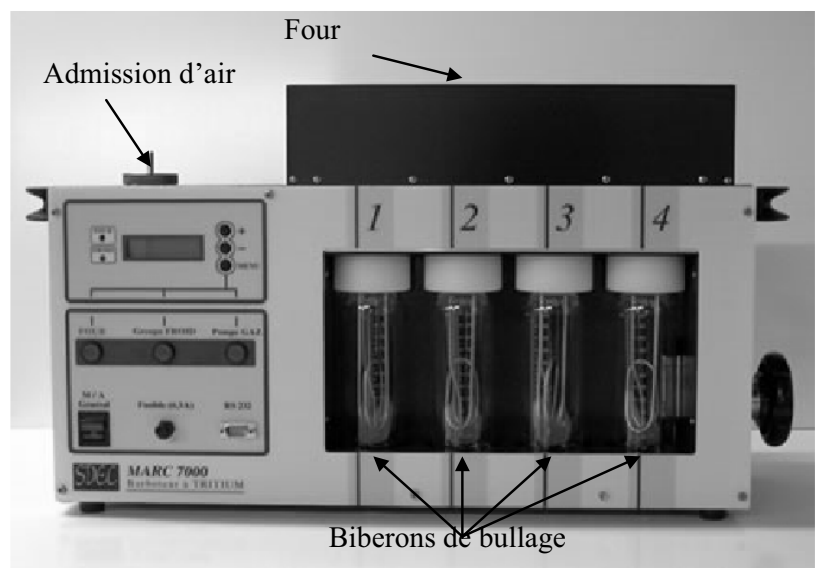

Figure 1 - MARC 7000. 
Le système de prélèvement d'air a été placé au plus proche du manipulateur de manière à estimer au mieux la totalité de l'air potentiellement inhalé durant l'ensemble des étapes de la manipulation.

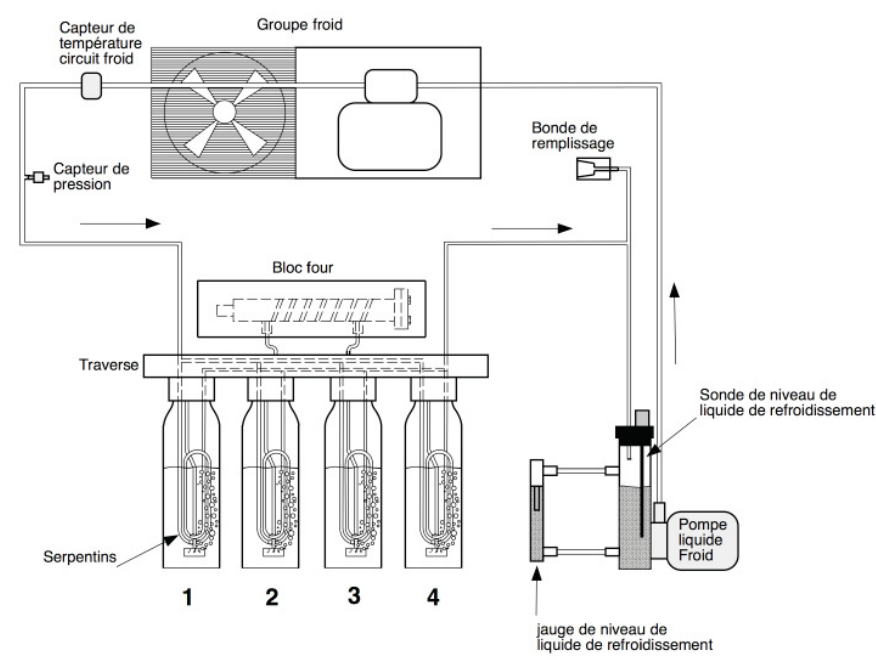

Synoptique du circuit froid

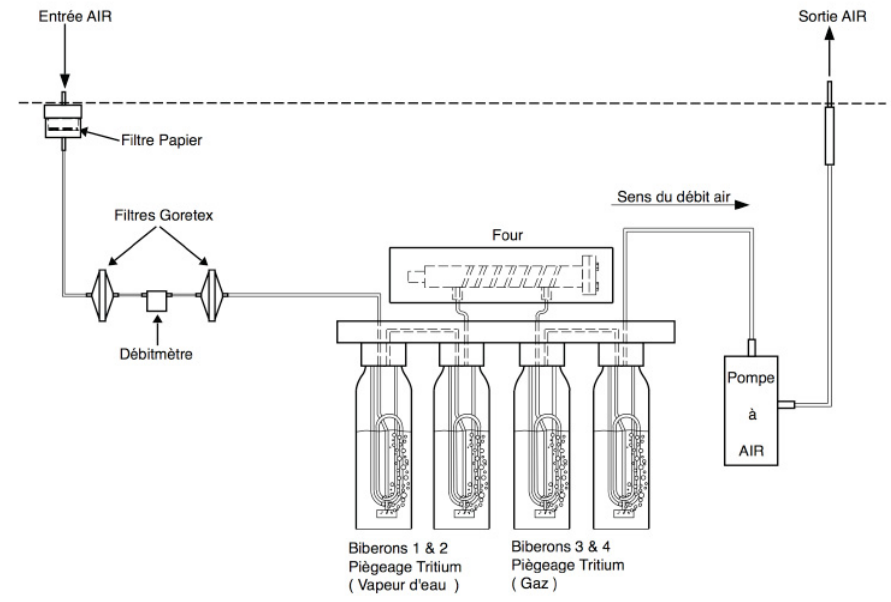

Synoptique du circuit d'air

Figure 2-Schémas de principe de fonctionnement des circuits froid et air.

Cooling system and air system schemes. 


\subsection{Protocole expérimental}

Pour chaque biberon de bullage, le volume total de $150 \mathrm{ml}$ est transféré dans des pots en plastique tarés. Cinq fioles de comptage sont réalisées par biberon ainsi que des fioles de référence associées. La fiole de référence est constituée de $10 \mathrm{ml}$ d'eau déminéralisée mélangée à $10 \mathrm{ml}$ de liquide scintillant.

Une fiole de comptage est constituée de $10 \mathrm{ml}$ (prélevés parmi les $150 \mathrm{ml}$ d'eau déminéralisée contenue dans chaque biberon) mélangée à $10 \mathrm{ml}$ de liquide scintillant (Ultima Gold ${ }^{\circledR}$ Low Level Tritium ou UGLLT, Perkin Elmer). Le temps de comptage est de 10 minutes par fiole. Les résultats sont exprimés en DPM (désintégrations par minute).

\subsection{Méthode de calcul}

Pour calculer l'activité A en tritium d'un biberon, on effectue les calculs suivants :

A = moyenne DPM (5 fioles d'échantillons) - moyenne DPM (5 fioles de référence) ramenée au volume réel dans le biberon et exprimée en $\mathrm{Bq}$.

La limite de quantification a été établie à 1,5 fois la valeur de DPM référence d'environ 30 DPM.

On ramène ensuite les activités totales des 4 biberons au volume d'air aspiré par le barboteur. L'activité est exprimée soit en $\mathrm{Bq}$ (pour le volume d'air aspiré par le barboteur) soit en en Bq. $\mathrm{m}^{-3}$.

On estimera également la valeur de dose résultante en Sv, pour un temps de travail de 2000 heures et un quotient respiratoire humain moyen de $1,2 \mathrm{~m}^{3} \cdot \mathrm{h}^{-1}$.

\section{Résultats des études de recherche de contamination atmosphérique}

Analyses de poste retenues sur 2 sites de l'Institut de recherche Pierre Fabre.

\subsection{Centre de recherche fondamentale}

La finalité de cet établissement est la recherche de nouvelles substances chimiques utilisables en médecine humaine principalement utilisés dans des indications thérapeutiques liées au système nerveux central (douleur chronique, dépression et anxiété, Alzheimer, schizophrénie...). Les radionucléides sous forme de sources non scellées sont utilisés pour des tests de binding liaison ligand froid - ligand chaud / récepteur in vivo et in vitro, binding fonctionnel in vitro, la recherche d'inhibiteurs 
d'enzymes, des dosages radio-immunologiques (RIA), de l'autoradiographie ou imagerie bêta.

Les radionucléides actuellement utilisés sont ${ }^{3} \mathrm{H},{ }^{14} \mathrm{C},{ }^{35} \mathrm{~S},{ }^{125} \mathrm{I}$ et ${ }^{33} \mathrm{P}$.

Les protocoles de manipulation dans le cas du tritium ont été sélectionnés en fonction des critères suivants :

- les quantités maximales mises en œuvre pour un même radionucléide ;

- les manipulations présentant des étapes sensibles (chauffage, utilisation d'agents chimiques, ouverture d'un incubateur à $37^{\circ} \mathrm{C}$, sacrifice des souris...) ;

- les manipulations les plus fréquentes ;

- les manipulations dont la durée d'exposition du manipulateur est la plus importante.

L'ensemble des zones réglementées de ce site sont ventilées avec un taux de renouvellement horaire de 10 . Les manipulations font parfois appel à plusieurs ligands tritiés.

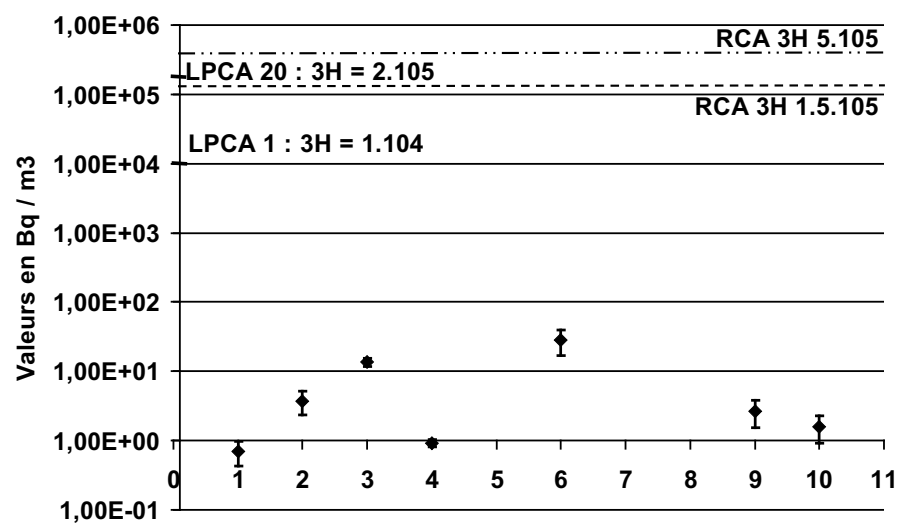

Référence des manipulations

Figure 3 - Analyse du risque d'exposition interne : ${ }^{3} \mathrm{H}$. Risk analysis of internal ${ }^{3} \mathrm{H}$ exposure.

Valeurs calculées sur une base de 2000 heures de travail par an.

Calcul de dose résultante : $A_{\text {inh }}=A v \times Q \times t$ puis $E_{\text {int }}=A_{\text {inh }} \times h(g)_{\text {inhalation }}$

Avec $Q=$ débit respiratoire humain moyen, généralement fixé à $1,2 \mathrm{~m}^{3} \cdot \mathrm{h}^{-1}$, on obtient alors une valeur de dose résultante $\left(E_{\text {int }}\right)$ en $\mathrm{Sv}$ ou en $\mu \mathrm{Sv}$. 


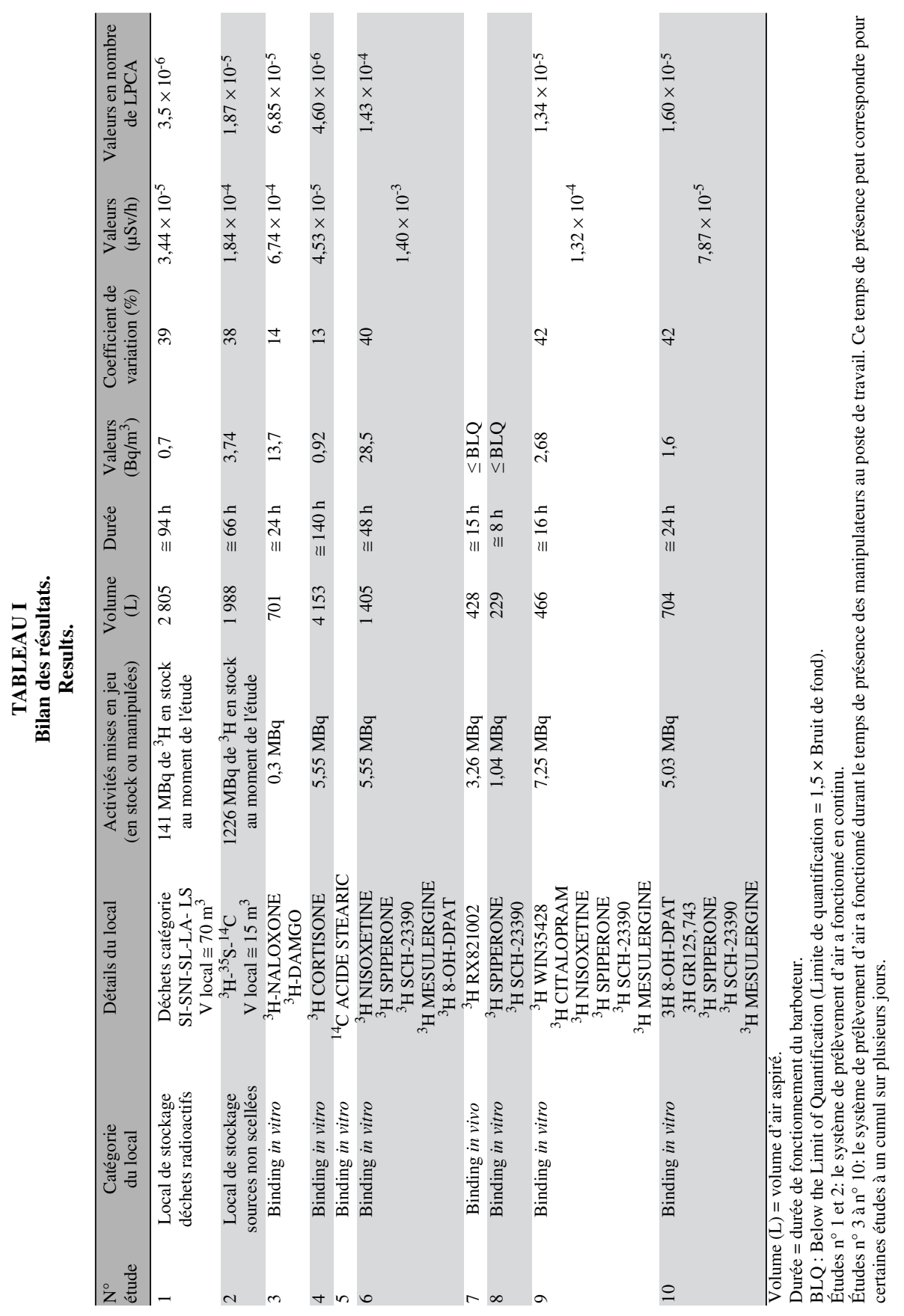




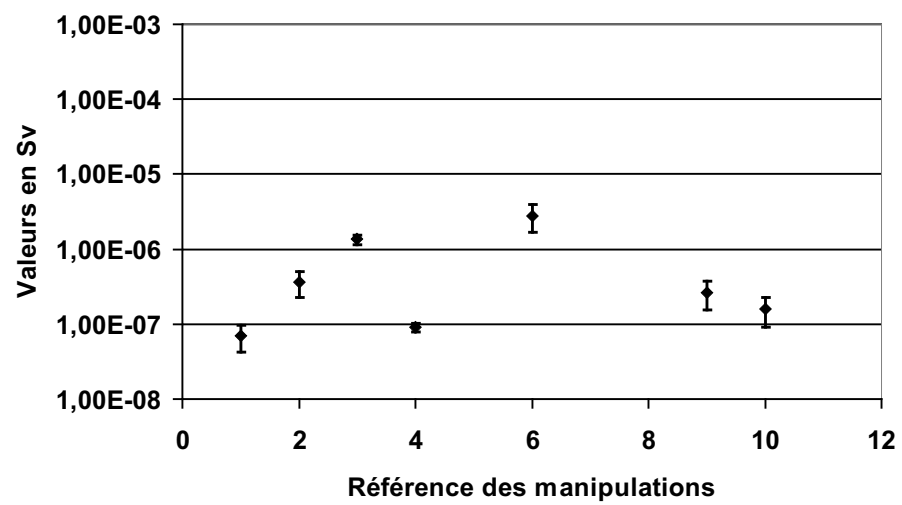

Figure 4 - Doses résultantes pour le tritium.

Tritium doses.

Dans les figures 3 et 4, les valeurs importantes d'écart-type observées sont directement liées aux faibles valeurs de DPM détectées en scintillation liquide, proches de la limite de quantification (de l'ordre de 30 DPM).

Les valeurs de doses résultantes obtenues sont très faibles et largement inférieures à la limite de la zone publique (soit un rapport de l'ordre de 1000 pour la majorité des manipulations).

L'analyse des résultats confirme que pour l'ensemble des situations analysées aucune ne relève d'une contamination atmosphérique significative.

Des résultats similaires ont également été obtenus au Centre d'immunologie ainsi que sur le centre de recherche et développement de Toulouse.

Globalement, on peut en déduire une valeur de facteur $K$ largement inférieure à celle proposée dans le Guide pratique "Radionucléides et Radioprotection » (Delacroix et al., 2006) avec passage de la valeur de 0,01 à une valeur de 0,0001 . Cette nouvelle valeur du facteur de volatilité du tritium aboutit à des expositions individuelles en adéquation avec les résultats urinaires négatifs.

\subsection{Centre expérimental et pharmacocinétique}

Le Centre expérimental et pharmacocinétique est constitué de trois départements : Cinétique préclinique, Pharmacologie générale et Toxicologie principalement.

Les radionucléides utilisés $\left({ }^{3} \mathrm{H}\right.$ et $\left.{ }^{14} \mathrm{C}\right)$ pour les tests in vitro et in vivo sont des produits commercialisés ou sont issus de synthèse à façon pour les molécules «Pierre Fabre ». 
L'ensemble des manipulations est soumis au Comité d'ethique animale et bénéficie d'un environnement « Bonnes pratiques de laboratoires » (BPL).

De la même manière que sur le site précédent, des situations estimées les plus pénalisantes pour le manipulateur ont été retenues pour cette étude.

Lors des analyses de poste, les résultats d'exposition interne obtenus avec un facteur $K=0,01$ étaient proches des limites réglementaires.

Par ailleurs, ces valeurs étaient également en contradiction avec la surveillance médicale renforcée ponctuelle des manipulateurs délivrant des résultats de radiotoxicologie urinaire toujours négatifs.

Détail de l'étude de «Local Lymph Node Assay » $\left(\right.$ LLNA $\left.^{1}\right)$

\section{$\underline{\text { Solution d'administration au laboratoire : }}$}

- activité : $37 \mathrm{MBq}$ dans un solvant aqueux ;

- préparation et contrôle sous sorbonne ;

- barboteur : aspiration durant environ $3 \mathrm{~h}$ avec tuyau fixé sur le bas de vitre de la sorbonne.

Phase expérimentale animale radioactive dans le module d'hébergement:

- activité : $37 \mathrm{MBq}$;

- traitement des différents groupes de souris : environ $1 \mathrm{~h}$ d'injection intraveineuse (veine caudale) de ${ }^{3} \mathrm{H}$-thymidine, sur paillasse ;

- hébergement : après leur traitement, les 4 souris/groupe sont hébergées dans des cages « cristal » à couvercle percé. Ces cages sont entreposées, au fil des traitements, dans un grand coffre. À la fin des traitements, ce coffre est fermé par un couvercle non ventilé pendant $5 \mathrm{~h}$;

- barboteur : aspiration environ $1 \mathrm{~h}$ avec tuyau fixé au plus près du manipulateur (phase de traitement) et environ $6 \mathrm{~h}$ dans le coffre abritant les cages de souris. Le taux de renouvellement d'air du local est de l'ordre de 16 volumes/h.

Au bout de $5 \mathrm{~h}$ après administration des souris, le couvercle du coffre est retiré, par les techniciens d'étude, dans le module d'hébergement. Les ganglions lymphatiques des souris sont ensuite prélevés pour les besoins de l'étude.

1 Local Lymph Node Assay (LLNA) : méthode qui évalue le pouvoir sensibilisant des substances chimiques et des mélanges. La définition d'un résultat positif dans le LLNA signifie une augmentation par prolifération de la cellule ganglionnaire qui devient, au moins trois fois plus grande que celle d'un contrôle.

Le test du LLNA est réalisé chez la souris et utilisé en toxicologie afin de définir le pouvoir sensibilisant des produits testés. 
TABLEAU II

Résultats obtenus à l'aide des barboteurs. Results obtained by bubbling.

\begin{tabular}{|c|c|c|c|c|c|c|c|}
\hline Phase d'étude & $\begin{array}{c}\text { Activité } \\
\text { départ } \\
\text { mise en jeu } \\
(\mathrm{MBq})\end{array}$ & $\begin{array}{c}\text { Durée } \\
\text { de } \\
\text { barbotage }\end{array}$ & $\begin{array}{l}\text { Volume } \\
\text { d'air } \\
\text { aspiré } \\
\text { (L) }\end{array}$ & $\begin{array}{c}\text { Activité } \\
\text { totale } \\
\text { barboteurs } \\
(\mathrm{KBq})\end{array}$ & $\begin{array}{c}\text { Contamination } \\
\text { atmosphérique } \\
(\%)\end{array}$ & $\begin{array}{c}\text { Exposition } \\
\text { interne } \\
\left(\mu \mathrm{Sv} \cdot \mathrm{h}^{-1}\right)\end{array}$ & $\begin{array}{c}\text { Exposition } \\
\text { interne } \\
\left(\text { nb LPCA }{ }^{*}\right)\end{array}$ \\
\hline $\begin{array}{c}\text { LLNA_ } \\
\text { Préparation } \\
\text { Solution } \\
\text { d'administration } \\
\text { ( }{ }^{3} \mathrm{H} \text {-thymidine) }\end{array}$ & 37 & $\cong 3 \mathrm{~h}$ & 83 & 0 & 0 & 0 & 0 \\
\hline $\begin{array}{l}\text { LLNA_Phase } \\
\text { expérimentale } \\
\text { radioactive }\end{array}$ & 37 & $\cong 7 \mathrm{~h}$ & 190 & 1,6 & 0,04 & $\begin{array}{c}\cong 0,4 \\
\text { Soit } \cong 800 \\
\mu S v \cdot \text { an }^{-1} \\
\text { pour } 2000 \mathrm{~h} \\
\text { de travail }\end{array}$ & 0,04 \\
\hline $\begin{array}{l}\text { Local déchets } \\
\text { radioactifs }\end{array}$ & 7,7 & $\cong 138 \mathrm{~h}$ & 4015 & $\cong 0$ & $\cong 0$ & 0 & 0 \\
\hline
\end{tabular}

* $:$ LPCA $=2 \mathrm{E} 5$ Bq.m ${ }^{-3}$ (Delacroix et al., 2006).

Limite zone surveillée : $7,5 \mu \mathrm{Sv} \cdot \mathrm{h}^{-1}$.

Limite zone contrôlée : $25 \mu \mathrm{Sv} \cdot \mathrm{h}^{-1}$

Globalement, on peut en déduire une valeur de facteur $K$ largement inférieure à celle proposée dans le Guide pratique "Radionucléides et Radioprotection » (Delacroix et al., 2006) avec passage de la valeur de 0,01 à une valeur de 0,0001. Cette nouvelle valeur du facteur de volatilité du tritium aboutit à des expositions individuelles en adéquation avec les résultats urinaires négatifs.

\section{Interprétation et discussion}

\subsection{Interprétation}

Malgré une certaine variabilité des résultats obtenus due à des valeurs proches de la limite de quantification (de l'ordre de $30 \mathrm{DPM}$, soit 1,5 $\times \mathrm{Bf}$ ), les valeurs de doses potentiellement inhalables obtenues sur les 2 sites restent très en dessous des valeurs autorisées par la réglementation.

Une étude similaire a également été menée avec le carbone 14 aboutissant à des valeurs de doses résultantes nulles, même lors d'étapes sensibles, comme l'évaporation d'une solution mère éthanolique sous courant d'azote.

Au Centre expérimental et pharmacocinétique, les résultats obtenus montrent une valeur d'exposition interne non négligeable pour la phase animale de l'étude LLNA, alors qu'aucune contamination atmosphérique n'est à déplorer pour la 
phase de préparation de la solution d'administration de thymidine tritiée. Le local de stockage des déchets radioactifs ne présente aucune contamination atmosphérique significative, le barboteur étant en marche pendant une semaine, de façon continue.

Afin de s'assurer que la contamination atmosphérique révélée par le barboteur est due à l'expiration des souris et non pas à une remise en suspension directe dans l'air de la thymidine tritiée, une étude complémentaire a été menée. Cette étude portant sur le métabolisme pulmonaire de la thymidine tritiée menée chez la souris nous permettra de chiffrer la contamination atmosphérique consécutive à une étude « LLNA ».

\section{2. Étude de l'excrétion pulmonaire d'eau tritiée par « métabowl»}

La solution d'administration utilisée est la même que celle préparée pour l'étude du pouvoir sensibilisant par la méthode LLNA. Après administration de la thymidine tritiée par voie intraveineuse, 10 souris sont placées dans un système de verrerie, en circuit fermé, appelé « métabowl » : les souris inspirent librement l'air apporté par une pompe et les gaz expirés sont piégés dans 2 «barboteurs» réfrigérés +1 flacon de garde. L'eau tritiée éventuellement expirée est alors recueillie pendant un temps déterminé $(1 \mathrm{~h}$ ou $5 \mathrm{~h})$. À l'issue de ce temps, correspondant à la durée de la phase animale de l'étude «LLNA », l'eau ultrapure des pièges est récoltée en vue d'un comptage du tritium par scintillation liquide.

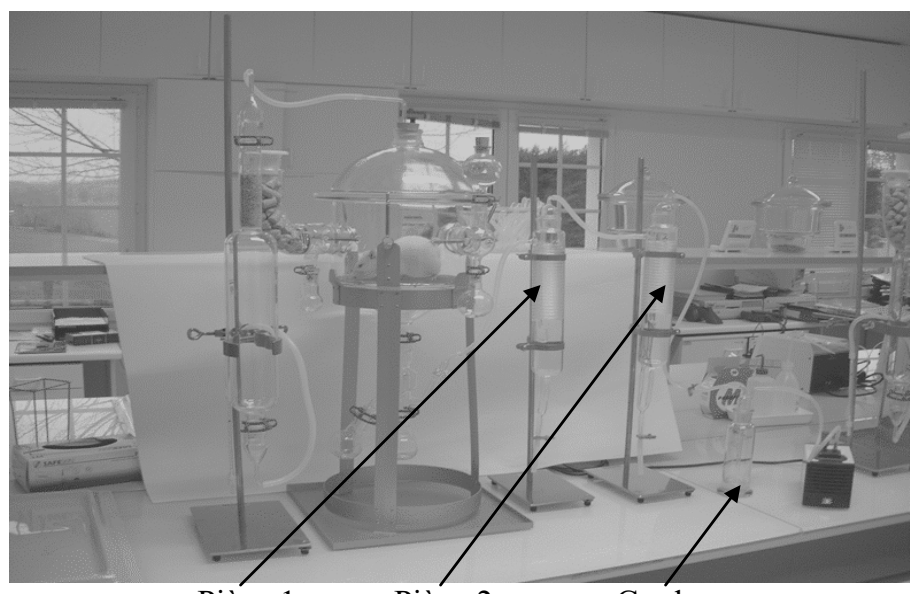

Piège 1 Piège 2 «Garde »

Figure 5 - Photo de "métabowl » hébergeant un rat. Rat exposure devices. 


\begin{tabular}{ccccccc} 
Résultats & \multicolumn{7}{c}{ TABLEAU III } \\
Résultats. \\
Results.
\end{tabular}

\section{Bilan de l'étude d'excrétion pulmonaire}

Même en considérant la situation la plus pénalisante, très éloignée de la réalité :

- nombre d'animaux maximum possible pour une étude ;

- techniciens d'étude inhalant la totalité du tritium expiré par les souris en $5 \mathrm{~h}$;

- ventilation du module non prise en compte $\left(R>16 \mathrm{~h}^{-1}\right.$ en réel) ;

- nombre maximum d'études réalisables par an.

On obtient :

- une classification du personnel (techniciens d'étude) : catégorie B $\left(0,13 \mathrm{mSv} \mathrm{an}^{-1}\right)$ (voir 5.3) ;

- une classification du local (module d'hébergement souris) : zone surveillée $(2,6 \mu \mathrm{Sv}$ sur $1 \mathrm{~h})$.

Soulignons le fait que ces valeurs sont à considérer de façon très prudente (car largement surestimées), sans perdre de vue qu'elles ne reflètent en aucun cas une contamination interne réelle des manipulateurs mais qu'elle donne une idée maximale de la contamination atmosphérique (au regard des hypothèses pénalisantes de calculs cités ci-dessus) qu'induit la phase d'étude in vivo considérée. 
Suite à ces résultats de l'étude de LLNA et toujours dans le souci de respect du principe ALARA, nous avons engagé les actions suivantes quant à la phase animale :

- communication des résultats au personnel concerné ;

- mise en place de grilles d'aération au niveau du coffre destiné à l'entreposage des cages de souris traitées à la thymidine tritiée ;

- déplacement du bac coffre vers les grilles d'aspiration du module d'hébergement.

\subsection{Impact sur le zonage et le classement du personnel}

Tous les locaux des différents centres de recherche Pierre Fabre où sont manipulés des radionucléides sont actuellement classés Zone surveillée avec passage des zones de stockage (sources mère et déchets) de Zone contrôlée à Zone surveillée.

Il faut noter que les activités des travailleurs peuvent varier rapidement dans les laboratoires de recherche, et par conséquent, la prise en compte d'une marge de sécurité est recommandée. De plus, bien que le niveau d'exposition individuelle, trouvé dans nos laboratoires, autorise à considérer les manipulateurs comme «non exposés », l'entreprise a fait le choix de maintenir les dits manipulateurs en catégorie $\mathrm{B}$. De ce fait, l'ensemble du personnel utilisant la radioactivité bénéficie au minimum d'un suivi médical renforcé avec surveillance radiotoxicologique urinaire.

Outre l'ensemble de ces mesures, un soin tout particulier a été apporté à la formation du personnel sur l'ensemble des sites Pierre Fabre, notamment lors de la réalisation des travaux pratiques (le temps d'enseignement alloué à cette partie a été augmenté avec davantage de mise en situation accidentelle ou incidentelle...).

\section{Conclusions}

En définitive, l'approche expérimentale nous a permis une évaluation des expositions internes lors des manipulations radioactives et ainsi la validation de nos analyses de poste.

Les résultats de nos études sont très rassurants puisqu'aucune contamination atmosphérique significative n'a été mise en évidence, excepté lors de l'administration de ${ }^{3} \mathrm{H}$-thymidine chez l'animal (contamination atmosphérique due à un métabolisme pulmonaire de la molécule et non pas à la volatilité du tritium dans des conditions normales de manipulation sur plan de travail). 
Les différentes actions engagées dans les laboratoires nous ont permis d'optimiser les conditions de travail du manipulateur permettant ainsi l' abaissement du risque de contamination atmosphérique à un niveau très faible et acceptable.

Ainsi, les résultats de ces études de contamination atmosphérique nous autorisent sur chaque site du Groupe Pierre Fabre à :

- valider le choix de la formule de calcul de dose résultante (voir 2.2);

- valider notre hypothèse de départ, à savoir une surestimation du facteur de volatilité $K$;

- utiliser une valeur du facteur $K$ égale à 0,0001 en cohérence d'une part avec les résultats expérimentaux et d'autre part avec nos conditions de travail.

Il faut cependant adopter une grande vigilance lors de la manipulation du tritium, selon ses différentes formes physico-chimiques, car le risque d'inhalation, aussi faible soit-il, reste inévitable tant que la mise en œuvre n'est pas réalisée en système hermétiquement clos.

Il en découle une question importante qui reste soumise à la réflexion : jusqu'où doit-on optimiser la radioprotection de nos travailleurs au nom du principe ALARA alors même que nous sommes en présence de valeurs de LPCA déjà très faibles ?

De même, nous souhaiterions compléter cette étude par d'autres procédés de détection applicables aux radioisotopes présents dans le domaine de la recherche.

Les données présentes dans ce document sont des résultats spécifiques pour des molécules données, dans des conditions expérimentales données. Il revient à chaque chef d'établissement, avec le concours de la PCR, de déterminer la nature et l'ampleur du risque dûes aux rayonnements ionisants pour d'autres molécules dans des conditions de mise en œuvre différentes.

Remerciements. Nous remercions la société SDEC pour les informations fournies sur le barboteur MARC 7000.

Nous remercions également $S$. Bréand et $S$. Tardif du service Informatique de Recherche des laboratoires Pierre Fabre.

\section{RÉFÉRENCES}

Baglan N., Ansoborlo E., Cossonet C., Fouhal L., Deniau I., Mokili M., Henry A., Fourré E., Olivier A. (2010) Métrologie du tritium dans différentes matrices : cas du tritium organiquement lié (TOL), Radioprotection 45, 369-390. 
Belot Y., Métivier H., Roy M. (1996) Le tritium : de l'environnement à l'homme (Collection IPSN). EDP Sciences.

Delacroix D., Guerre J.-P., Leblanc P. (2006) Guide pratique Radionucléides et Radioprotection (Collection Radioprotection). EDP Sciences.

LNE-LNHB/CEA (2006) Table des radionucléides.

Radecki J.J., Aubert B., Cordier G., Fracas P. (2007) La mise en œuvre de l'arrêté zonage, Radioprotection 42, 463-476. 